\title{
Dramaturgie du réel, mettre en scène l'histoire
}

Kafka, Vanzetti, auteurs du Siècle d'or

\section{Luis Araújo}

\section{OpenEdition}

\section{Journals}

Édition électronique

URL : https://journals.openedition.org/cher/2164

DOI : $10.4000 /$ cher.2164

ISSN : 2803-5992

Éditeur

Presses universitaires de Strasbourg

\section{Édition imprimée}

Date de publication : 29 mai 2018

Pagination : 73-77

ISBN : 979-10-344-0019-5

ISSN : 1968-035X

\section{Référence électronique}

Luis Araújo, « Dramaturgie du réel, mettre en scène l'histoire », reCHERches [En ligne], 20 | 2018, mis en ligne le 01 décembre 2021, consulté le 02 décembre 2021. URL : http://journals.openedition.org/cher/ 2164 ; DOI : https://doi.org/10.4000/cher.2164

\section{(c) (i) (2)(2)}

Ce(tte) œuvre est mise à disposition selon les termes de la Licence Creative Commons Attribution Pas d'Utilisation Commerciale - Partage dans les Mêmes Conditions 4.0 International. 


\title{
Dramaturgie du réel, mettre en scène l'histoire Kafka, Vanzetti, auteurs du Siècle d'or
}

Luis Araújo 1

\begin{abstract}
T a réécriture de textes de provenances diverses donne à l'auteur contemporain Lla possibilité de confronter des faits de la réalité qui mettent en évidence les tensions et le devenir de nos sociétés, dans leurs fondements comme dans leur développement actuel et ce, tant si cette réalité appartient à une époque historique que si elle nous est contemporaine. Le travail que l'auteur entreprend sur un matériau préalable exige toujours une sélection, non seulement au niveau sémantique ou thématique; cette sélection ne porte pas uniquement sur des textes au sens strict mais également sur un lexique, des cadences rythmiques, une atmosphère à transposer vers le texte d'arrivée, dans le but d'assimiler de multiples facteurs stylistiques aptes à définir l'imaginaire à reproduire / partager avec le(s) texte(s) source(s).

Chacun sait que toute récupération de l'Histoire procède à une relecture de notre présent et que la réécriture d'événements contemporains équivaut à une réinterprétation du sens. Pourtant, à quelques notables et brillantes exceptions près, la relecture et réécriture des discours dans leur dimension stylistique, contextuelle, dans leur atmosphère particulière, selon un processus de construction partagé avec le texte source, dans la pragmatique de l'énonciation des discours - au-delà de la récupération plus ou moins fidèle de segments de textes à proprement parler - n'a été que peu étudié.

Le passage de textes sources provenant d'autres genres vers la dramaturgie entraîne un questionnement dont j'essaierai de suivre le fil au cours de cette réflexion.
\end{abstract}

1 Auteur et metteur en scène espagnol. 


\section{Questions dramaturgiques}

\section{Le personnage ${ }^{2}$}

Un personnage incarne une vision du monde. Il peut s'agir d'une attitude mentale, d'une idéologie, d'une quête, d'une conception de la vie ou des relations humaines. En fait, il s'agit de quelque chose de très différent d'une personne, ce n'est même pas l'imitation ou la représentation d'une personne. Un personnage est bien davantage un vecteur de force en action qui pousse et oriente l'action dans un sens déterminé. Il peut arriver qu'il se contredise, qu'il change, qu'il se transforme ou pas; cela dépend du réseau de relations qui s'établit avec de nombreux autres facteurs: l'action, les autres personnages/vecteurs/forces, l'osmose entre la réalité de la représentation (le public et son environnement réel) et la fiction représentée (la fable), le transfert entre scène et hors scène dans la réalité comme dans la fiction. Et sans doute beaucoup d'autres facteurs non mentionnés dans cette brève esquisse.

\section{Crescendo dramatique}

Je l'appelle ainsi par convention. En réalité, c'est une dynamique dans laquelle quelque chose doit croître ou décroître progressivement. Ce qui veut dire que la construction de ce que nous appelons traditionnellement la tension dramatique est un processus dans lequel:

- ou bien l'intensité croît et nous amène à des niveaux où l'émotion, la sensorialité, la lucidité intellectuelle, le paradoxe, etc., sont plus exacerbés.

- ou bien le processus décroît et s'étiole, provoquant une absence croissante, un manque progressif et par conséquent, à partir de quelque élément constitutif antérieur, provoque alors un douloureux tarissement émotionnel et intellectuel.

Dans les deux cas il s'agit, une fois de plus, d'un flux et d'un reflux d'énergie dont la mise en acte, en scène, provoque ces mêmes mouvements dans la communauté réunie dans la salle de spectacle.

\section{Rythme}

C'est la raison pour laquelle un rythme est absolument nécessaire. J'avoue ne pas savoir expliquer pourquoi. Bien sûr, nous savons tous que, depuis que nous avons connaissance de l'activité des hommes, les rituels ancestraux, les célébrations communautaires, la fête, se déroulent selon un rythme socialement accepté et partagé. Le théâtre, et par conséquent la dramaturgie qui le préfigure, évolue également selon un rythme partagé. J'aimerais ici faire une brève parenthèse. On pense généralement à la musique d'une époque pour représenter l'ambiance de cette époque. Cette approche illustrative ne me paraît avoir de

2 Je me permets de renvoyer ici à mon article «El estatuto múltiple de algunos de mis personajes» in: Nuevas Tendencias en el Teatro Hispánico del Siglo xxi, à paraître aux éditions Visor en Espagne. 
sens que si le flux énergétique proposé par la dramaturgie est bercé par le même rythme, mais il n'y a aucune raison qu'il en soit ainsi, j'espère l'avoir montré à travers la présentation des vecteurs et des éléments exposés jusqu'ici.

Le rythme marque le flux et le reflux d'énergie que la représentation transmet aux spectateurs en les faisant participer de façon empathique à ce mouvement. Et je voudrais préciser ici que lorsque je parle d'empathie, je ne renvoie pas seulement au courant émotionnel ou sentimental mais plutôt à une empathie psychosensorielle qui implique autant la capacité intellectuelle du spectateur que sa capacité sensorielle et/ou émotionnelle.

\section{Climax}

Lorsque nous, les artisans du théâtre, parvenons à faire que cela se produise, nous atteignons ce que les théoriciens cherchent depuis très longtemps sous le vocable de "théâtralité». C'est pourquoi, je me risquerai à affirmer que le transfert vers la dramaturgie de matériaux non dramatiques, historiques, réels, livresques, équivaut à la construction artisanale d'un texte contenant tous ces aspects de sorte qu'il permette à une équipe (une compagnie, terme dans lequel il convient d'englober tous les artisans intervenants dans le spectacle) de produire sur scène ce courant énergétique créateur d'empathie dans les trois sens que j'ai indiqués auparavant (peut-être dans davantage encore, les termes que j'emploie n'étant absolument pas exhaustifs).

\section{Représentabilité}

À ce stade, nous pouvons nous demander ce qui est ou n'est pas représentable. Non pas au sens du mimétique, ni même au sens de l'iconique, mais au sens de la théâtralité, de ce qui est susceptible de créer ces mouvements d'énergie empathique.

La spécificité du langage théâtral passe par cette capacité communicative et si la condition indispensable de la présence physique des interprètes/ performeurs est réaffirmée jusqu'à plus soif, c'est parce que la présence physique rend possible, comme nul autre moyen, ce transfert empathique entre les personnes. Si nous ajoutons à cela, les caractéristiques particulières attachées à la réunion publique, et on me pardonnera ce qui suit, de même que l'absence d'une médiatisation, qu'elle soit technologique ou instrumentale, c'est-à-dire, la transmission directe entre personnes, dans laquelle les corps et/ou les voix sont les instruments prioritaires, nous aurons l'essence même de la communication scénique (théâtre, danse, chant...), spécifiquement différente de tant d'autres médias, non seulement technologiques comme l'audiovisuel en général, mais également instrumentaux, orchestraux ou empruntant diverses modalités en rapport avec les arts plastiques. Je soutiens que là est la dramaturgie.

Ce qui n'exclut pas l'intervention, le complément, l'appui de tous les médias instrumentaux et technologiques que l'on souhaite utiliser au service de cette transmission empathique de personne à personne, privilégiant cette transmission qui constitue le drame en soi. Toute substitution de cet élément 
central et essentiel par n'importe quelle instrumentation ou médiation technique entraîne une dérive vers des conceptions dont la valeur artistique et créative ne me semble pas susceptible d'être remise en question. C'est la théâtralité du spectacle qui l'est. Une succession d'images de corps maquillés, habillés, éclairés, mis en musique peut à mon sens donner un résultat splendide dans le domaine des arts plastiques, et je pourrais goûter avec un énorme plaisir ce spectacle; ce que je remets en question, c'est sa "dramaticité». La création d'images, en soi, ne relève pas du drame, même si son contenu s'avère «dramatique», car elle ne rend pas possible l'échange, le crescendo.

\section{Qu'est-ce qui est «dramatisable»?}

Ainsi, les contenus à dramatiser dans l'histoire sont ceux où le temps disparaît et où, comme dans la suspension du temps décrite par Proust, le passé et le présent sont une et même chose hors du temps. Vanzetti, tête de turc dans la lutte féroce pour éradiquer le syndicalisme aux États-Unis; Kafka, torturé par son besoin de solitude et d'isolement pour pouvoir écrire et, pourtant, amoureux et désireux de vivre son amour, les monarques espagnols de la maison d'Autriche tentant, par leurs grandes réalisations architecturales, d'entrer dans la postérité de l'Histoire, n'existent-ils pas aujourd'hui parmi nous?

\section{Quels matériaux, quels référents?}

Ceux qui affectent fondamentalement les questions profondes de notre connaissance existentielle, de notre éthique, de notre capacité de survie, de notre capacité de réalisation, notre bonheur et notre plaisir. Et d'une manière générale ceux qui remettent en question notre capacité de compréhension du monde et de la réalité. Des conflits intrinsèques.

\section{Quel discours? Quel langage?}

Le discours de l'opposition, de l'agôn, du paradoxe, de l'impuissance face à la toute-puissance, du savoir et de la compréhension face à l'ignorance, de la raison face au pouvoir, de l'humanité face à la force brute.

Un langage pour chaque discours, non un langage réaliste mais plutôt celui qui crée le flux d'énergie de ce discours. Celui qui véhicule l'émotion et l'argumentation des pôles en conflit toujours entrelacés, ni univoque ni unidirectionnel: biunivoque et dans la mesure du possible, multiréférentiel, associatif, ouvert à la métaphore, à la lecture multiple, à l'intertextualité, à l'encyclopédie de la vie et de l'art, aux connaissances vécues et apprises.

Et ensuite l'atmosphère, le climat, les notions en rapport avec l'ouverture ou la réclusion, la luminosité ou les ombres, l'activité ou le statisme, l'inertie ou la résistance. Les gradations entre tous ces facteurs construisent la dynamo énergétique du texte, sa virtualité théâtrale ou «théâtralisable».

Les récentes découvertes des neurosciences nous disent clairement que notre cerveau compose une vision cohérente de la réalité à partir de données isolées et éparses qu'il réunit, combine et met en relation. Je vais donc me permettre 
d'affirmer que notre conception de ce que nous appelons la réalité, incognoscible ébullition de la matière extérieure à chacun de nous, pourrait bien être, en réalité, une très intéressante dramaturgie créée par notre cerveau.

\section{Bibliographie}

Réécritures par Luis Araújo

La parte contratante, (Cabaret littéraire, à partir de nombreux auteurs), Revue Escena, no 29, avril 1996, Barcelone.

Carlota o la noche mejicana, (version espagnole de l'original de Lilliane Wouters), Revue Tramoya, no 37, oct.-déc. 1993, Université de Veracruz (Mexique).

Vanzetti, (à partir des lettres de Vanzetti et des actes du procès Sacco et Vanzetti), - Primer Acto, no 254, mars 1994, Madrid.

- coll. «Biblioteca Antonio Machado de Autores Contemporáneos», Ed. Visor, Madrid, 1996.

- Contemporary Spanish Plays, Ed. Estreno, New Brunswick (New Jersey), 1999 (en anglais).

La construcción de la catedral, (à partir de «El Antiguo Régimen: los Reyes Católicos y los Austrias", tomo III, Historia de España, Madrid, Alfaguara, 1973), Aide à la création de la Consejería de Cultura de la Comunidad Autónoma de Madrid, Premio internacional Tramoya pour le meilleur texte théâtral en langue espagnole de l'année 2000.

- Revista Tramoya, no 65, oct.-déc. 2000, Université de Veracruz (Mexique)

- El Juego Eterno. Teatro de Luis Araújo, Ed. Fundamentos, Madrid, 2001 (junto con [avec] «Prototipo de poniente»).

- Emilio Coco, Teatro spagnolo contemporaneo. I giovani drammaturghi, vol. III, Edizioni dell'Orso, Alessandria, 2004 (Italie).

Prototipo de poniente, (à partir de «Memorias del Futuro y Diarios de las estrellas», Stanislav Lem), EL Juego Eterno: Teatro de Luis Araújo, Ed. Fundamentos, Madrid, 2001 (junto con [avec] «La construcción dela catedral»).

¿Quien nos robó la música?, (à partir des actes des procès contre les responsables de la dictature Argentine et leur suivi dans la presse), Résidence d'auteur: récupération de la Mémoire Historique du Centre de détention de La Perla, Córdoba (Argentine). Inédit. Dramaturgie pour un ballet muet.

Kafka enamorado, (à partir des lettres et journaux de Franz Kafka), Autores en el Centro, no 8, Centro Dramático Nacional. INAEM, Ministerio de Educación Cultura y Deporte. Madrid, 2013.

No perdáis este tren, (à partir de La Mère de Maxime Gorki) in Vallejo, Araújo, López Llera, Tres comedias de miedo, Francisco Gutiérrez Carbajo, CÁTEDRA, Letras Hispánicas, Madrid, 2016. 